\title{
Differential effects of lesions of the dorsomedial and dorsolateral caudate-putamen on reaction time performance in rats
}

\author{
Wolfgang Hauber ${ }^{b} *$, Werner J. Schmidt ${ }^{\text {a }}$ \\ "University of Tühingen. Zoological Institute, Department of Neuropharmacology', Mohlstr. 54/1, D-72074 Tühingen, German! \\ ${ }^{n}$ University of Stuttgart. Biological Institute. Department of Animal Physiology. Pfaffenwaldring 57. D-70550 Stuttgart. Germam!
}

(Received 16 June 1993; revised 5 November 1993: accepted 5 November 1993)

\begin{abstract}
In order to investigate the role of the dorsomedial and dorsolateral caudate-putamen $(\mathrm{CPu})$ in movement initiation of rats, we examined the effects of quinolinic acid lesions $(30 \mathrm{nmol}$ in $1 \mu 1)$ in these striatal subregions in a simple reaction time task. Results show that lesions of the dorsomedial, but not of the dorsolateral $\mathrm{CPu}$ increased reaction times. These findings provide further evidence for a functional heterogenity of the $\mathrm{CPu}$ and demonstrate an involvement of the dorsomedial $\mathrm{CPu}$ in processes related to rapid initiation of responses.
\end{abstract}

Key words: Reaction time; Locomotor initiation; Dorsomedial caudate-putamen; Dorsolateral caudate-putamen; Striatal heterogeneity; Rat

There is now considerable anatomical, biochemical and behavioural evidence for a functional differentiation of the striatum [8] and distinct cortico-striato-thalamo-cortical loops may exist which process different aspects of behaviour [1]. These findings are primarily based on primate studies, however in the rat brain there may exist at least in part a similar organization. Several rodent studies showed that the medio-lateral organization of the caudateputamen $(\mathrm{CPu})$ is behaviourally relevant, since lesions of the lateral $\mathrm{CPu}$ specifically produced motor deficits $[17,18]$ while lesions of the medial $\mathrm{CPu}$ impaired delayed alternation and discrimination learning $[7,10,18,22]$. Lateral and medial CPu seem to mediate different behavioural functions and these functions are closely related to those mediated by the anatomically linked regions of the cortex: the lateral $\mathrm{CPu}$ and the anatomically related sensorimotor cortical regions have been suggested to form a loop mediating motor functions. In contrast, the medial $\mathrm{CPu}$ receives prominent glutamatergic projections from the medial prefrontal cortex. This loop has been implicated in processing cognitive, i.e. complex, non-motor-functions [20].

In the present study we investigated the effects of lesions in the dorsomedial and dorsolateral $\mathrm{CPu}$ on reaction time

* Corresponding author. Fax: (49) (711) 685-5096. performance of rats in a task demanding rapid locomotor initiation. According to the loop model it was expected that lesions in these subregions should differentially affect reaction time performance. In fact it was recently shown that the relative contribution of the medial and lateral $\mathrm{CPu}$ to performance of a visual reaction time task is distinct [3]. We used lesion placements in the dorsomedial and dorsolateral $\mathrm{CPu}$ which are not exactly in the same anterio-posterior plane. According to anatomical studies the medial prefontal cortex projects primarily to the more rostral parts of the medial $\mathrm{CPu}$, while the sensorimotor cortex mainly projects more caudally to the lateral $\mathrm{CPu}$ $[2,14]$. Therefore, the dorsomedial lesion was placed more rostrally than the lateral lesion.

Male Sprague-Dawley rats (Interfauna, Tuttlingen, FRG) were used weighing 250-275 $\mathrm{g}$ at the beginning of the experiment. They were housed in groups of four or five animals with free access to water. Food was restricted to $12 \mathrm{~g}$ standard maintenance diet (Altromin, Lage, FRG) per animal and day. Rats were housed in a room maintained at a constant temperature $\left(22 \pm 3^{\circ} \mathrm{C}\right)$ on a $12: 12$ light-dark cycle (lights on $6.00 \mathrm{~h}$ ).

The experiments were performed in a modified runway made of transparent perspex as described previously [9,11]. Briefly, the apparatus consisted of a start box $(18 \times 9 \times 9 \mathrm{~cm})$ and a runway $(100 \times 9 \times 9 \mathrm{~cm})$ terminating 
in a goal box $(22 \times 9 \times 9 \mathrm{~cm})$. The entrance to the runway was lockable by a remote controlled guillotine door situated between start box and runway. The entrance to the runway was monitored by an infrared photocell beam (IDEC, FRG; resolution: < $10 \mathrm{~ms}$ ) horizontally mounted directly behind the guillotine door. A combined stimulus light $(10 \mathrm{~W})$ and tone $(8 \mathrm{kHz}, 40 \mathrm{~dB})$ signalled the simultaneous opening of the front door (duration of the opening: $<80 \mathrm{~ms}$ ). The stimulus lights, one on each side, and a loudspeaker were located in front of the start box. A control unit synchronized stimulus presentation and opening of the guillotine door. Control unit and photoelectronic switch were connected with an oscilloscope (ITT-metrix OX 750/2, Nürnberg, FRG) and a printer for evaluating the latencies between stimulus presentation and photobeam interruption.

The rats were trained for rapid initiation of locomotion in response to the stimulus to receive a food reward (food pellet $45 \mathrm{mg}$; Noyes, Lancaster, UK): a food-deprived rat was placed into the start box facing the closed guillotine door blocking the entrance to the runway. After a variable delay $(3-10 \mathrm{~s})$ the stimulus signalled the simultancous
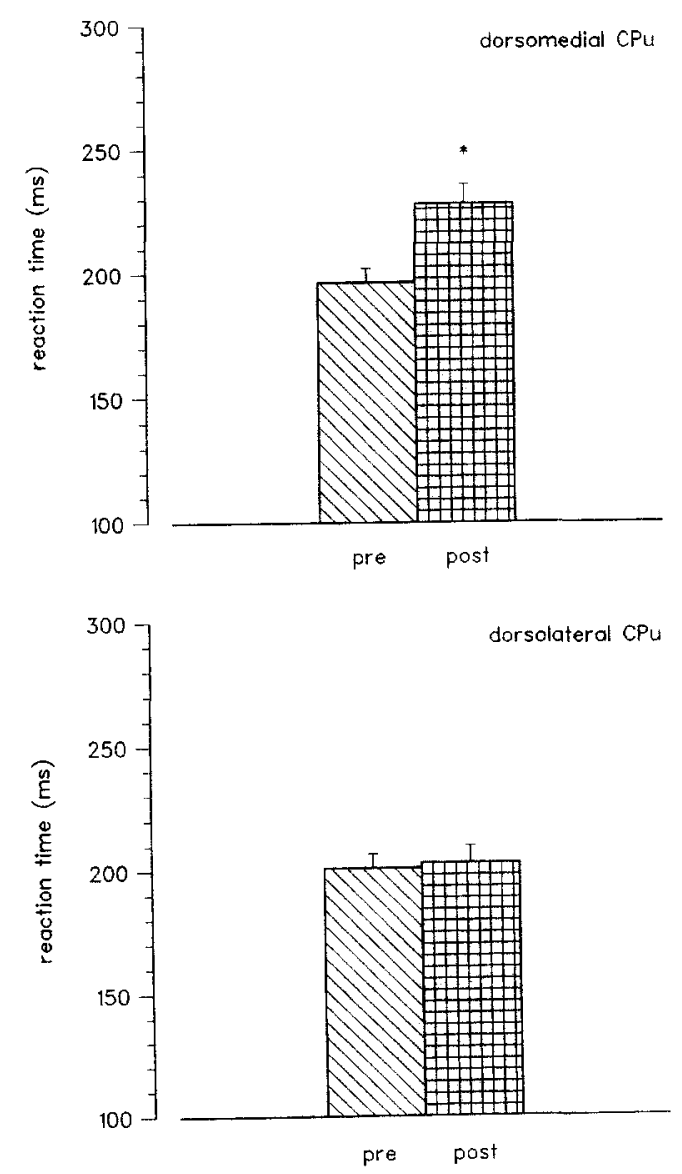

Fig. 1. Mean pre- and postoperative reaction times $( \pm$ S.E.M.) of animals with quinolinic acid lesions in the dorsomedial $\left(N=7, n_{\text {preap }}=270\right.$; $\left.n_{\text {postor }}=270\right)$ or dorsolateral $\left(N=7 ; n_{\text {preep }}=257 ; n_{\text {bostop }}=279\right) \mathrm{CPu}$. $* P<0.001$ (Student's' $t$-test). opening of the front door. A trained rat rapidly initiated locomotion, moved through the runway to the goal box and received the food reward in a baited cup. When the pellet was eaten or $10 \mathrm{~s}$ passed the rat was placed again into the start box for a new trial. The reaction timo of locomotor initiation was defined as latency from stimulus presentation up to photobeam interruption. The effect of an animals' prestart position relative to the guillotine door on reaction time measures was negligible because of the narrow dimensions of the start box and the fact that trained rats always look a prestart position close to the guillotine door. First of all, rats were habituated individually to the baited apparatus for $10 \mathrm{~min}$. From the following day on. animals were trained in one session per day, Each session comprised 10 successive trials. After the rats had learned the task ( 10 correct trials, i.c. with reaction times between $100-1000 \mathrm{~ms}$ ) the preoperative performance was tested in 4 sessions. Thereafter. animals received stereotaxic lesions. The postoperative performance was tested after it recovery period of 7 days. The reaction times from correct trials in pre- and postoperative sessions were pooled and subjected to Student's $t$-test. The data are presented as means and standard error of the mean ( \pm S.E.M.). Surgery was performed under sodium pentobarbilal anaesthesia $(50 \mathrm{mg} / \mathrm{kg}$. . i.p.) with atropinsulfate pretreatment (0.25 mg/kg, i.p.; Serva, Heidelberg, FRG). Rats were placed in a Trent Wells stereotaxic instrument. Quinolinic acid (Sigma, Deissenhofen, FRG) $30 \mathrm{nmol}$ in $1 \mu 10.1 \mathrm{M}$ phosphate buffer, pH 7.0) or vehicle ( $1 \mu 10.1 \mathrm{M}$ phosphate buffer, $\mathrm{pH} 7.0$ ) was infused via a 26 gauge cannula at a constant rate over $10 \mathrm{~min}$. The cannula was left in place for 2 min after the infusion. Lesions in the dorsomedial or dorsolateral subregion of the $\mathrm{CPu}$ were made at the following placements (with reference to bregma): dorsomedial CPu. anterio-posterior: $+2 \mathrm{~mm}$, dorsolateral $2.0 \mathrm{~mm}$, ventral $5.0 \mathrm{~mm}$; dorsolateral $\mathrm{CPu}$ anterio-posterior +0.9 mm, dorsolateral $3.5 \mathrm{~mm}$, ventral $5.5 \mathrm{~mm}$ (according to the atlas of Paxinos and Watson [16]). At the end of lesting all animals were anaesthetized with sodium pentobarbital $(50 \mathrm{mg} / \mathrm{kg}$, i.p.) and transcardially perfused with $0.9^{\circ}$, saline followed by a $4 \%$, solution of phosphate butfered formalin ( $\mathrm{pH}$ 7.4). Brains were removed, postfixed and thereafter stored in $30 \%$, sucrose solution. Coronal sections of $40 \mu \mathrm{m}$ were cut with a ryostat (Reichert\& Jung. Nußloch, FRG) and stained with Cresyl violet. The scctions were examined for qualitative assessment of neuronal damage. The areas of neuronal loss from different anterio-posterior planes were mapped on brain sections according to the atlas of Paxinos and Watson [16].

Quinolinic acid lesions within the dorsomedial $\mathrm{CPu}$ produced a small but significant increase of reaction time $(t=3.27, \mathrm{df}=538 ; P<0.001)($ Fig. 1$)$. In contrast, lesions of the dorsolateral $\mathrm{CPu}$ did not affect reaction time per- 
Table 1

Mean correct reaction times $( \pm$ S.E.M.) of animals with vehicle infusions into the dorsomedial or dorsolateral caudate-putamen

\begin{tabular}{llll}
\hline Site & Preoperative $(\mathrm{N}, \mathrm{n})$ & Postoperative $(\mathrm{N}, \mathrm{n})$ & $\boldsymbol{t}$ \\
\hline Medial & $207.4 \pm 6.8 \mathrm{~ms}(5: 197)$ & $211.4 \pm 4.3 \mathrm{~ms}(5: 194)$ & 0.50 \\
Lateral & $191.5 \pm 3.8 \mathrm{~ms}(4 ; 159)$ & $199.7 \pm 4.0 \mathrm{~ms}(4: 155)$ & 0.62 \\
\hline
\end{tabular}

formance $(t=0.223, \mathrm{df}=534, P=0.82)$ as shown in Fig. 1 . $V$ chicle infusions also did not change reaction times postoperatively (Table 1). The histological examination revealed that quinolinic acid infusion produced a circumscribed neuronal cell loss (Figs. 2 and 3). Within this area a moderate gliosis was found while ventricular alterations or a striatal shrinkage were not detected by gross inspection. Furthermore the overlap of lesions within the dorsolateral and dorsomedial $\mathrm{CPu}$ was minimal. Control brains with vehicle infusions showed no degenerative alterations except a gliosis along the canula track.

In accordance with a previous report [3] these results demonstrate that reaction time was affected selectively by lesions of the dorsomedial $\mathrm{CPu}$. Quinolinic acid is a potent neuroexcitotoxin acting via $N$-methyl-D-aspartate receptors. The dose of quinolinic acid used here was shown to be the treshold dose for producing consistent neuronal degeneration of a defined size without affecting fibres of passage [4,5]. Numerous neurochemical and histochemical studies showed that quinolinic acid predominantly

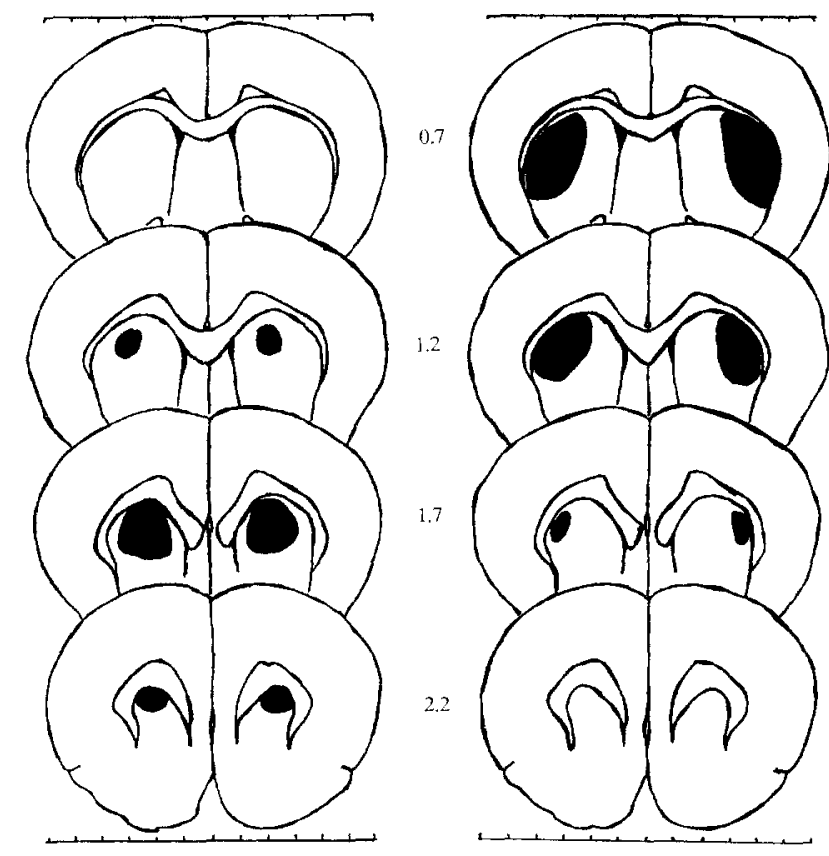

Fig. 2. Location and extent of quinolinic acid-induced lesions of the dorsolateral (right) and dorsomedial (left) caudate-putamen in different anterio-posterior planes of striatum (according to the atlas of Paxinos and Watson [16]). Abscissae are scaled in mm. Numbers represent the anterio-posterior planes with reference to bregma. destroys medium spiny neurons with gamma-aminobutyric acid (GABA) as neurotransmitter leaving aspiny neurons with somatostatin unaffected [6]. Thercfore quinolinic acid-induced impairments seen here are most probably due to the loss of striatal GABAergic projection neurons bearing NMDA receptors.

In a recent study [3] rats with unilateral ibotenic acid lesions were tested in a reaction time task demanding head movements in response to visual stimuli. In this task rats with lesions of the medial CPu showed a significant slowing of initiation latency to a similar extent as observed here, while lesions of the lateral CPu had no effects on reaction time. The present data corroborate and extend these findings in that irrespective of the kind of movement and the type of neuroexcitotoxin used, and, despite of minor differences in the lesions placements and different lesion modes (bilateral versus unilateral), lesions of the dorsomedial $\mathrm{CPu}$ impaired rapid initiation of responses triggered by a stimulus. An involvement of the caudate nucleus and the putamen in externally and internally generated processes which are related to the initiation of behavioural acts was also shown in primate studies [15,21].

However, our data give no indications about the underlying mechanisms resulting in an increase of reaction time. A disrupted attentional control as previously suggested [3] may also account for the slowing of reaction time seen here. Evidence for an impaired attentional control came, among others, from studies using maze tasks. For instance, pharmacological blockade of output neurons in the dorsomedial $\mathrm{CPu}$ impaired acquisition of delayed alternation in a T-maze possibly due to attentional deficits [10]. This and numerous other findings are in line with a role of the medial $\mathrm{CPu}$ in processing complex behavioural functions. In accordance with the loop model it was further found that 6-hydroxydopamine lesions in the medial prefrontal cortex, the region which sends prominent projections to the medial $\mathrm{CPu}$, produced a marked increase of reaction times in the task used here [12]. Thus the cognitive cortico-striato-cortical loop may play a role in monitoring reaction time performance. On the other hand the failure to detect an involvement of the dorsolateral $\mathrm{CPu}$ in control of reaction time was unexpected in view of the motor functions of this striatal subarea [13]. However the detection of a reaction time impairment is dependent on the task and stimulus configuration used and other 

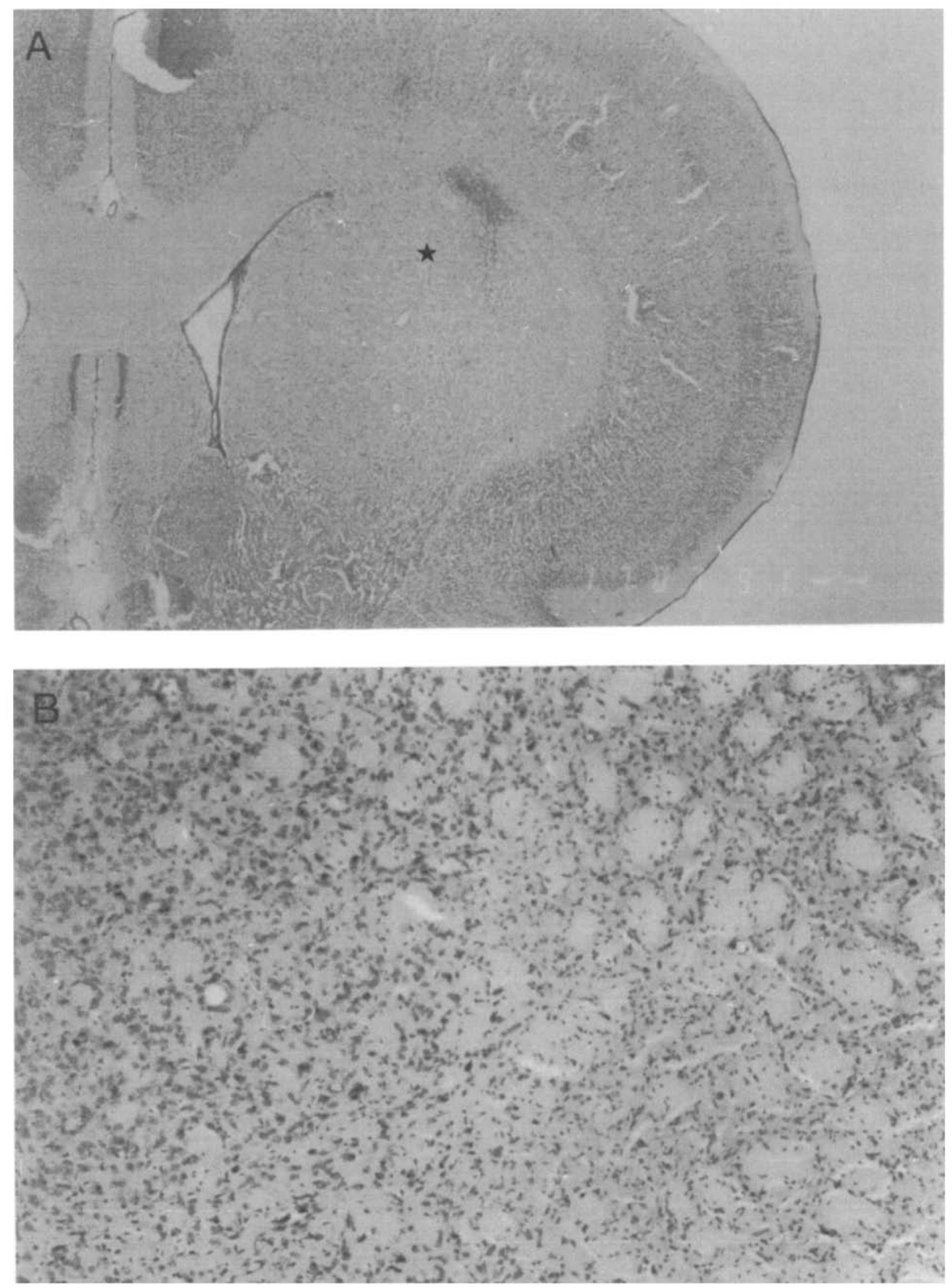

Fig. 3. Micrographs showing an example of the quinolinic acid injection site in the dorsolateral striatum. A: location of the injection as indicated by the cannula track (magnification $8 \times$ ). B shows at higher magnification $(32 \times)$ a part of the borderline between intact (left side) and damaged tissue (right side) illustrated in A. Note the numerous cell bodies staned with Cresyl violet on the left side which are absent on the right side.

factors as e.g. the dose of the neurotoxin. In fact in a recent study using a visual spatial discrimmination task it was suggested that the lateral striatum is the principle area concerned with control of reaction time although the medial CPu also plays a role [19]. It seems therefore that both striatal subareas contribute to intact reaction time performance. With regard to the different functional loops of which both subregions are part of, the underlying mechanisms disrupted after lesions in the dorsomedial and dorsolateral CPu leading to a slowing of reaction time are probably different, but they are not very well characterized.
In summary the present study provides further evidence for a functional heterogenity of the CPu and demonstrates an involvement of the dorsomedial $\mathrm{CPu}$ in processes related to the rapid initiation of responses.

We thank E. Wacker and S.Schmidt for excellent technical assistance. The study was supported by the Deutsche Forschungsgemeinschaft (SFB 307/A4).

\section{References}

[1] Alexander, G.E., DeLong. M.R. and Strick, P.L., Parallel organization of functionally segregated circuits linking basal ganglia and cortex, Anu Rev. Neurosci., 9 (1986) 357-381 
[2] Berendse, H.W., Galis-de Graaf, Y. and Grocnwegen, H.J., Topographical organization and relationship with ventral striatal compartments of prefrontal corticostriatal projections in the rat, $J$. Comp. Neurol., 316 (1992) 314-347.

[3] Brown, V.J. and Robbins, T.W., Elementary processes of responsc sclection mediated by distinct regions of the striatum. $J$. Neurosci.. 9 (1989) $3180-3186$.

[4] Churchill, L.. Dilts, R.P. and Kalivas, P.W., Changes in gammaaminobutyric acid, $\mu$-opioid and neurotensin receptors in the accumbens-pallidal projection after discrete quinolinic acid lesions in the nucleus accumbens, Brain Res., 511 (1990) 41-54.

[5] Davies, S.W. and Roberts, P.J.. Sparing of cholinergic neurons following quinolinic acid lesions in the rat striatum, Neuroscience, 26 (1988) 387-393.

[6] Difiglia, M., Excitotoxic injury of the neostriatum: a model for Huntington's disease, Trends Neurosci, 13 (1990) 286-289.

[7] Divac. I., Markowitsch, H.J. and Pritzel, M., Behavioural and anatomical consequences of small intrastriatal injections of kainic acid in the rat. Brain Res., 151 (1978) 523-532.

[8] Goldman-Rakic. P.S. and Selemon, L.D., New frontiers in basal ganglia research, Trends Neurosci., 13 (1990) 241-243.

[9] Hauber, W., A novel reaction time task for investigating force and time parameters of locomotor initiation in rats, Experientia, 46 (1990) 1084-1087.

[10] Hauber. W. and Schmidt, W.J., Effects of intrastriatal blockade of glutamatergic transmission on the acquisition of $\mathrm{T}$-maze and radial maze tasks, J. Neural. Transm., 78 (1989) 29-41.

[11] Hauber. W. and Schmid1, W.J., The NMDA antagonist dizocilpine (MK-801) reverses haloperidol-induced movement initiation deficits, Behav. Brain Res., 41 (1990) 161-166.

[12] Hauber. W. Bubser, M. and Schmidt W.J., Cortico-striatal loops and reaction time perormance in rats, Eur. J. Neurosci., 4 (1991) 167.

[13] Hauber, W. and Schmidt, W.J., Discrete quinolinic acid lesions of the lateral but not of the medial caudate-putamen reversed haloperidol-induced catalepsy in rats, J. Neural. Transm., in press.

[14] McGeorge, A.J. and Faull, R.L.M., The organization of the projection from the cerebral cortex to the striatum in the rat, $\mathrm{Neu}$ roscience, 29 (1989) 503-537.

[15] Montgomery, E.B. and Buchholz, S.R., The striatum and motor cortex in motor initiation and execution. Brain Res., 549 (1991) $222-229$

[16] Paxinos, G. and Watson, C. The Rat Brain in Stereotaxic Coordinates, Academic Press, Sydney, 1986.

[17] Pisa, M., Motor somatotopy in the striatum of rat: manipulation, biting and gait, Behav. Brain Res., 27 (1988) 21-35.

[18] Pisa, M. and Cyr, J., Regionally selective roles of rat's striatum in modality-specific discrimination learning and forelimb reaching, Behav. Brain Res., 37 (1990) 281-292.

[19] Pretsell, D.O. and Robbins, T.W.. Simple and choice reaction time performance following unilateral ibotenic acid lesions of dorsomedial or dorsolateral striatum in the rat. Soc. Neurosci. Abstr.. (1991) 483.7 .

[20] Robbins, T.W. and Brown, V.J., The role of the striatum in the mental chronometry of action: a theoretical revicw, Rev. Netrosci., 2 (1990) 181-213.

[21] Schultz, W. and Romo, R., Neuronal activity in the monkey striatum during initiation of movements, Exp. Brain Res., 71 (1988) $431-436$.

[22] Wikmark, R.G.E., Divac, I. and Weiss, R., Retention of spatial delayed alternation in rats with lesions in the frontal lobes. Brain Behav. Evol., 8 (1973) 329-339. 